\title{
Notas nomenclaturais em Salacioideae (Celastraceae)
}

\author{
Nomenclatural notes on Salacioideae (Celastraceae)
}

Julio Antonio Lombardi ${ }^{1}$

\begin{abstract}
Resumo
Sãopropostas para Salacioideae (Celastraceae) sul-americanas quatronovas combinações:Peritassamyrsinoides (A.C.Sm.) Lombardi,P. nectandrifolia (A.C.Sm.) Lombardi,P. petiolata (A.C.Sm.) Lombardie Tontelea passiflora (Vell.)Lombardi. Palavras-chave: Brasil, Celastraceae, Peritassa, Tontelea.

\section{Abstract}

Four new combinations are proposed for the following South American Salacioideae (Celastraceae): Peritassa myrsinoides (A.C.Sm.) Lombardi, P. nectandrifolia (A.C.Sm.) Lombardi, P. petiolata (A.C.Sm.) Lombardi, and Tontelea passiflora (Vell.) Lombardi.
\end{abstract}

Key words: Brazil, Celastraceae, Peritassa, Tontelea.

\section{Introdução}

Reconhecida anteriormente como táxon distinto, a família Hippocrateaceae atualmente é considerada parafilética e incluída em Celastraceae como duas subfamílias: Hippocrateoideae, com 19 gêneros e ca. 100 espécies, e Salacioideae, com seis gêneros e ca. 260 espécies (Simmons 2004), com distribuição em áreas tropicais e subtropicais do mundo.

Os limites genéricos são controversos nas Hippocrateaceae, sendo reconhecidos de dois, Salacia L. e Hippocratea L. (Peyritsch 1878), a até 25 gêneros (Simmons 2004). Aqui é adotado o conceito mais recente, reconhecendo quatro gêneros para as Salacioideae neotropicais: Cheiloclinium Miers, Peritassa Miers, Salacia e Tontelea Miers (Smith 1940; Mennega 1997; Simmons 2004).

Finalizando o checklist das Celastraceae para o projeto Lista de Espécies da Flora do Brasil, faz-se necessário a proposição de novas combinações, como abordado no presente estudo.

\section{Material e Métodos}

Neste artigo é seguida a divisão genérica e em subfamílias de Simmons (2004). As conclusões foram baseadas pela análise de exsicatas, fotografias e coleções de espécimes-tipo dos herbários: A, BM, F, G, GH, K, M, MICH, NY, P, S, US, W (siglas conforme Thiers 2009).

\section{Resultados e Discussão}

Peritassa myrsinoides (A.C.Sm.) Lombardi, comb. nov. Tontelea myrsinoides A.C.Sm., Brittonia 3: 499. 1940. Salacia myrsinoides (A.C.Sm.) J.F.Macbr., Field Mus. Nat. Hist., Bot. Ser. 13 (3A, 1): 214. 1951. Tipo: PERU. LORETO: Mishuyacu, near Iquitos, XII.1929, fl., G. Klug 712 (holótipo NY!; isótipos F! (FNeg 68510), US!).

Peritassa nectandrifolia (A.C.Sm.) Lombardi, comb. nov. Salacia nectandrifolia A.C.Sm., Bull. Torrey Bot. Club 66: 241. 1939. Tontelea nectandrifolia (A.C.Sm.) A.C.Sm., Brittonia 3: 497. 1940. Tipo: BRASIL. AMAZONAS: São Paulo de Olivença, basin of creek Belem, basin of Rio Solimões, 26.X-11.XII.1936, fl., B.A. Krukoff 8788 (holótipo NY!; isótipos BM!, F! (FNeg 40890), G!, $\mathrm{K}$ !, MICH!, P!, S!).

Peritassa petiolata (A.C.Sm.) Lombardi, comb. nov. Salacia petiolata A.C.Sm., Bull. Torrey Bot. Club 66: 242. 1939. Tontelea petiolata (A.C.Sm.) Mennega, Novon 2: 234. 1992. Tipo: BRASIL. AMAZONAS: basin of Rio Juruá, near mouth of Rio Embira (tributary of Rio Tarauaca), 30.VI.1933, fl., B.A. Krukoff 5141 (holótipo NY!; isótipos A!, G!).

As três espécies precedentes, incluídas por Smith (1940) no gênero Tontelea, não apresentam

${ }^{1}$ UNESP - Universidade Estadual Paulista, Instituto de Biociências de Rio Claro, Depto. Botânica, Av. 24-A 1515, 13506-900, Rio Claro, SP, Brasil. cissus@rc.unesp.br 
as apomorfias do último gênero, ou seja, disco unido aos ângulos do ovário por septos e estigma 3-lobado, exceto em T. cylindrocarpa (A.C.Sm.) A.C.Sm., com estigma pontual.

Os discos curto-tubulares e anteras oblongas, com deiscência transversa ou transverso-apical, e conectivo inconspícuo, indicam que provavelmente são melhores posicionadas no gênero Peritassa, possivelmente junto ao grupo de espécies constituído por P. calypsoides (Cambess.) A.C.Sm., P. glabra (A.C.Sm.) Lombardi, P. longifolia Lombardi, P. mexiae A.C.Sm. e P. sadleri Lombardi, que compartilham estas características.

Tontelea passiflora (Vell.) Lombardi, comb. nov. Clercia passiflora Vellozo, Fl. flumin.: 30. 1829. Lectótipo aqui designado, Fl. flumin. icon. 1, tab. 74. 1831 .

Cuervea ovalifolia Miers, Trans. Linn. Soc. London 28: 371. 1872. Tontelea ovalifolia (Miers) A.C.Sm., Brittonia 3: 476. 1940. Salacia ovalifolia (Miers) J.F.Macbr., Field Mus. Nat. Hist., Bot. Ser. 13: 212. 1951. Tipo: COLÔMBIA. META: Villavicencio, 1851-1857, fl., J. Triana s.n. [3516] (holótipo K!; isótipos BM!, P!).

Salacia richardii Peyr. in Mart. \& Eichler, Fl. bras. 11(1): 148. 1878. Tontelea richardii (Peyr.) A.C.Sm., Brittonia 3: 478. 1940. Tontelea ovalifolia subsp. richardii (Peyr.) Görts \& Mennega, Novon 2: 233. 1992. Tipo: GUIANA FRANCESA. In sylvis ripariis Guyanna, 1791, fl., L.C.M. Richard s.n. (holótipo P!; isótipo P-JU! (12034)).

Salacia fluminensis Peyr. in Mart. \& Eichler, Fl. bras. 11(1): 149. 1878. Tontelea fluminensis (Peyr.) A.C.Sm., Brittonia 3: 477. 1940. Tipo: BRASIL. Sem localidade determinada (provavelmente Rio de Janeiro ou Minas Gerais): X.1823, fl., L. Riedel s.n. (holótipo M!; isótipos B† (FNeg 13354), F! (FNeg 62272), GH!, K!, P!, US!, W!).

Tontelea passiflora, aqui considerada sensu lato, engloba espécimes com estigma de lobos inteiros, alternados com os estames, folhas grandes, predominantemente não pontuadas na face abaxial, e frutos também grandes, elipsóides e de epicarpo espesso e lenhoso.

As dificuldades para a delimitação das espécies afins, Tontelea passiflora, T. fuliginea Lombardi e T. attenuata Miers, levou a uma série de interpretações bastante divergentes. Görts-van Rijn e Mennega (1994) incluiram T. fluminensis como sinônimo de T. ovalifolia, enquanto Lombardi (2006) considerou T. fluminensis distinta de T. ovalifolia, baseado principalmente no número de óvulos por lóculo e na presença de verrucosidade nas inflorescências.

Reavaliando esta posição anterior, acreditase que este grupo inclui três espécies com flores morfologicamente indistintas: Tontelea passiflora, $T$. fuliginea e $T$. attenuata, esta última com folhas geralmente mais estreitas, quase sempre conspicuamente pontuadas de negro na face abaxial, inflorescências glabras, não verrucosas, e frutos relativamente pequenos e esferóides, de epicarpo fino a espesso; $T$. fuliginea, com folhas largas, não pontuadas de negro na face abaxial, inflorescências pubérulas, não verrucosas, e frutos desconhecidos; e T. passiflora, com folhas também largas, muito raramente esparso-pontuadas de negro na face abaxial, inflorescências glabras ou verrucosas e frutos relativamente grandes, elipsóides, mais ou menos assimétricos, e de epicarpo espesso.

O número de óvulos por lóculo, de 4-6, notado por Smith (1940) e Lombardi (2006) como uma característica que distinguiria Tontelea fluminensis e T. ovalifolia, não foi confirmado nos exemplares da bacia amazônica e América Central; no que foi possível se observar, constatou-se a presença de dois óvulos por lóculo.

\section{Agradecimentos}

O autor agradece à Fapesp - Fundação de Amparo à Pesquisa do Estado de São Paulo (n ${ }^{\circ}$ 2005/00437-3) o financiamento recebido e ao CNPq - Conselho Nacional de Desenvolvimento Científico e Tecnológico, as bolsas de Produtividade em Pesquisa (n 523026/96-0, 300220/2003-0 e 306395/ 2006-1), e de Apoio Técnico (nº 502506/2005-9). Também aos revisores as críticas que contribuíram para a melhoria do manuscrito, e aos curadores dos herbários mencionados os empréstimos, envio de duplicatas e hospitalidade ao autor enquanto visitando suas instituições.

\section{Referências}

Görts-van Rijn, A.R.A. \& Mennega, A.M.W. 1994. Hippocrateaceae. Flora of the Guianas. Ser. A, Phanerogams. Vol. 16. Pp. 3-81.

Lista de Espécies da Flora do Brasil. 2010 [continuamente atualizada]. Celastraceae. Disponível em <http:// floradobrasil.jbrj.gov.br/2010/>. 
Lombardi, J.A. 2006. A new species of Tontelea from Amazonian Peru and Ecuador, and notes on the Tontelea attenuata species group (Celastraceae, Hippocrateoideae). Brittonia 58: 52-58.

Mennega, A.M.W. 1997. Wood anatomy of the Hippocrateoideae (Celastraceae). IAWA 18: 331368.

Peyritsch, J. 1878. Hippocrateaceae. In: Martius, C.F.P. \& Eichler, A.W. (ed.). Flora brasiliensis . Leipzig. Vol. 11, pars 1. Pp. 125-164.
Simmons, M.P. 2004. Celastraceae. In: Kubitzki, K. The families and genera of vascular plants. Vol. 6. Berlin. Pp. 29-64.

Smith, A.C. 1940. The American species of Hippocrateaceae. Brittonia 3: 341-555.

Thiers, B. 2009 [continuamente atualizado]. Index Herbariorum: A global directory of public herbaria and associated staff. New York Botanical Garden's Virtual Herbarium. Disponível em <http:// sweetgum.nybg.org/ih/>. Acesso em 22 julho 2009. 